\title{
Re: Lower Urinary Tract Dysfunction in Children. What Do Pre-School Teachers Know About It?
}

\author{
Patricia Lordelo, Fabio Maron, Daniela G. Barros, Danilo V. Barroso, Jose Bessa Jr, Ubirajara \\ Barroso Jr
}

Department of Pediatric Urology, School of Medicine, Federal University of Bahia, Salvador, Bahia, Brazil

Int Braz J Urol, 33: 383-388, 2007

To the Editor:

The authors devised a questionnaire that they administered to 50 pre-school teachers "to evaluate the basic knowledge of lower urinary tract dysfunction". Teachers of young children can have a significant impact in detecting children who may need urologic care. In addition, they may be, in part, responsible for reinforcing the voiding habits of these children, for better or for worse. Pre-school teachers in this study appeared to have mixed notions about what should be considered as normal and abnormal voiding behavior. It should not be surprising that there was little difference in responses based on the education or experience of the teachers, as this is generally not part of the formal training or continuing education of teachers.

The age range of children taught by these teachers may also serve to explain the discrepancies among the teacher's perceptions of what is normal.
Frequency in a 4-year-old child whose voiding habits are still immature probably warrants less attention than a 7-year-old with frequency. Such nuances in the development of urinary control only serve to reinforce the need to educate teachers of young children about proper voiding habits, as the authors have advocated.

Behavioral therapy can reduce both urinary tract infections and urinary incontinence in a significant number of children. Because children spend a good proportion of their waking hours at school, teachers can have a significant impact on this health issue by reinforcing proper voiding habits and alerting parents and healthcare professionals to those children at risk for clinically significant underlying urologic disease. Further studies demonstrating the effectiveness of educational programs for teachers are needed.

Dr. M. Chad Wallis

Assistant Professor of Surgery Division of Urology University of Utah School of Medicine Salt Lake City, Utah, USA E-mail: chad.wallis@hsc.utah.edu 